\title{
A NOTE ON THE ALTERNATING DIRECTION IMPLICIT METHOD FOR THE NUMERICAL SOLUTION OF HEAT FLOW PROBLEMS
}

\author{
JIM DOUGLAS, JR.
}

1. Introduction. In companion papers $[1 ; 6]$ recently Peaceman, Rachford, and the author introduced a finite difference technique called therein the alternating direction implicit method for approximating the solution of transient and permanent heat flow problems in two space variables. The validity of the method was established only in the case of a rectangular domain. Since then the procedure has been tested successfully on several more complex examples [4] without proof. The purpose of this short note is to prove in the case of non rectangular domains that the solution of the alternating direction method for the parabolic problem converges to the solution of the differential equation as the increments of the independent variables diminish in a proper manner, that the iterative adaptation for the elliptic problem converges to the solution of the Laplace difference equation, and to give an efficient choice of the parameter sequence involved in this iteration.

2. Parabolic problem. Let $D$ be an open, connected set in the plane bounded by a curve $C$ made up of closed polygons with sides parallel to the coordinate axes. Assume, moreover, that there exists a sequence $\left\{\Delta x_{\alpha}\right\}, \Delta x_{\alpha} \rightarrow 0$, such that, for each $\alpha, C$ coincides with a polygon through lattice points $\left(i \Delta x_{\alpha}, j \Delta x_{\alpha}\right)$ with sides parallel to the axes. The notation below is the same as in [1]. Consider the boundary value problem

$$
\begin{aligned}
\frac{\partial^{2} u}{\partial x^{2}}+\frac{\partial^{2} u}{\partial y^{2}} & =\frac{\partial u}{\partial t}, & (x, y) \in D, 0<t \leq T, \\
u(x, y, 0) & =f(x, y), & (x, y) \in D, \\
u(x, y, t) & =g(x, y, t), & (x, y) \in C, 0<t \leq T .
\end{aligned}
$$

The alternating direction implicit difference analogue of $(2.1)$ is the following:

$$
\begin{gathered}
\Delta_{x}^{2} w_{i, j, 2 n+1}+\Delta_{y}^{2} w_{i, j, 2 n}=\left(w_{i, j, 2 n+1}-w_{i, j, 2 n}\right) / \Delta t,(i \Delta x, j \Delta x) \in D, \\
\Delta_{x}^{2} w_{i, j, 2 n+1}+\Delta_{y}^{2} w_{i, j, 2 n+2}=\left(w_{i, j, 2 n+2}-w_{i, j, 2 n+1}\right) / \Delta t,(i \Delta x, j \Delta x) \in D, \\
w_{i, j, 0}=u_{i, j, 0},(i \Delta x, j \Delta x) \in D, w_{i, j, m}=u_{i, j, m},(i \Delta x, j \Delta x) \in C .
\end{gathered}
$$

Received by the editors September 17, 1955. 
The advantages of this system are discussed in [6].

We shall perform a stability analysis on (2.2) to enable the application of the convergence theorem of [2]. For this purpose it is sufficient to allow $w_{i, j, m}$ to vanish on $C$. Let $A$ be a matrix corresponding to the operator $\Delta_{x}^{2}, B$ one corresponding to $\Delta_{y}^{2}$, and $w_{m}$ the column vector with components $w_{i, j, m}$. Then, (2.2) can be written as:

$$
w_{2 m+2}=\left(B-\frac{1}{\Delta t}\right)^{-1}\left(A+\frac{1}{\Delta t}\right)\left(A-\frac{1}{\Delta t}\right)^{-1}\left(B+\frac{1}{\Delta t}\right) w_{2 m} .
$$

As $A$ and $B$ commute and are symmetric, it is easy to see that

$$
M=\left(B-\frac{1}{\Delta t}\right)^{-1}\left(A+\frac{1}{\Delta t}\right)\left(A-\frac{1}{\Delta t}\right)^{-1}\left(B+\frac{1}{\Delta t}\right)
$$

is symmetric. For, if $P$ and $Q$ are symmetric, $P Q$ is symmetric if and only if $P Q=Q P$, and $P Q=Q P$ if and only if $P Q^{-1}=Q^{-1} P$.

Stability of (2.2) is implied by having all of the eigenvalues of $M$ less than one in magnitude. First, in the case $D$ is a square, the eigenfunctions are known to be

$$
\phi_{p q}=\sin \pi p x_{i} \sin \pi q y_{i},
$$

and the eigenvalues to be $\left(M \phi_{p q}=\mu_{p q} \phi_{p q}\right)$

$$
\mu_{p q}=\frac{1-\lambda \sin ^{2}(\pi p \Delta x / 2)}{1+\lambda \sin ^{2}(\pi p \Delta x / 2)} \cdot \frac{1-\lambda \sin ^{2}(\pi q \Delta x / 2)}{1+\lambda \sin ^{2}(\pi q \Delta x / 2)},
$$

where $\lambda=4 \Delta t /(\Delta x)^{2}$. Thus, for any $\lambda>0,(2.2)$ is stable for a square region. The general case then follows from [5, p. 164, Theorem 3$]$ by enclosing $C$ in a square and removing points from the lattice one by one until the desired region is obtained.

Assume that the initial conditions and boundary condition are such that (2.1) possesses in the closed region $(x, y) \in D \cup C, 0 \leq t \leq T$, a solution $u$ with bounded $u_{x x x x}, u_{y y y y}, u_{x x t}, u_{y y t}$ and $u_{t t}$. Then, Theorem 2 of [2] may be applied to yield the following result:

THEOREM 1. Under the conditions on $D$ and $u$ stated above, the linearly interpolated solution $w(x, y, t)$ of (2.2) converges in the mean to $u(x, y, t)$ in $D \times[0, T]$. Moreover, the $L_{2}$ norm of $u(x, y, t)-w(x, y, t)$ is $O\left((\Delta x)^{2}+(\Delta t)^{2}\right)$.

If, in addition to the above hypotheses, $g(x, y, t)$ vanishes identically, then the argument of [3] can obviously be adapted to yield a convergence theorem in the $L_{2}$ topology for variable time steps. In fact, it is easy to see that the error is $O(\Delta x)^{2}$ if the time steps satisfy the relation 


$$
\frac{\Delta t_{n}}{(\Delta x)^{2}}=a+b t_{n}
$$

where $\Delta t_{n}=t_{n+1}-t_{n}, t_{0}=0$.

3. Elliptic problem. Consider in a region $D$ as described in $\$ 2$ the first boundary value problem for the potential equation:

$$
\begin{aligned}
\frac{\partial^{2} u}{\partial x^{2}}+\frac{\partial^{2} u}{\partial y^{2}} & =0, & & (x, y) \in D, \\
u(x, y) & =g(x, y), & (x, y) & \in C .
\end{aligned}
$$

It is well known [5] that under quite general conditions the solution of the Laplace difference equation

$$
\begin{aligned}
\Delta_{x}^{2} w_{i, j}+\Delta_{y}^{2} w_{i, j} & =0, & & (i \Delta x, j \Delta x) \in D, \\
w_{i, j} & =g_{\imath, j}, \quad & & (i \Delta x, j \Delta x) \in C,
\end{aligned}
$$

converges to $u$ as $\Delta x \rightarrow 0$. We shall not be interested here in this theoretical problem but in the practical problem of obtaining the solution of the linear equations in (3.2) for some fixed $\Delta x$.

Let $w_{i j}^{(0)}$ be an arbitrary lattice function agreeing with $g_{i j}$ on $C$, and define $w_{i j}^{(n)}$ iteratively as follows:

$$
\Delta_{x}^{2} w_{i j}^{(2 n+1)}+\Delta_{y}^{2} w_{i j}^{(2 n)}=\frac{w_{i j}^{(2 n+1)}-w_{i j}^{(2 n)}}{a_{n}}, \quad(i \Delta x, j \Delta x) \in D
$$

$$
\begin{aligned}
\Delta_{x}^{2} w_{i j}^{(2 n+1)}+\Delta_{y}^{2} w_{i j}^{(2 n+2)} & =\frac{w_{i j}^{(2 n+2)}-w_{i j}^{(2 n+1)}}{a_{n}}, & & (i \Delta x, j \Delta y) \in D, \\
w_{i j}^{(m)} & =g_{i j}, & & (i \Delta x, j \Delta x) \in C .
\end{aligned}
$$

Now, the eigenfunctions occurring in the expansion of $w_{i j}-w_{i j}^{2 n}$ are the same as those appearing in the treatment of the parabolic case for $\Delta t=a_{n}$. Thus, for any $a_{n}>0$ each two-step iteration reduces the magnitude of the coefficient of each eigenfunction. While this implies the convergence of $w_{i j}^{(m)}$ to $w_{i j}$ for fixed $a_{n}=a$, this choice of the sequence of parameters $a_{n}$ is not the most efficient.

It was found [6] that for a square region of side $a$ that taking a cycle of iterations with

$$
\lambda_{n}=\frac{4 a_{n}}{(\Delta x)^{2}}=\left(\frac{1-R}{1+R}\right)^{2 n} / \sin ^{2} \frac{\pi \Delta x}{2 a}, \quad n=0, \cdots, m,
$$

where $\lambda_{m-1}>1, \lambda_{m} \leq 1$, reduces each coefficient by a factor of $R$ at 
least. Thus, to reduce each coefficient by a factor of $\exp (-Q)$ requires at most $Q /-\log R$ cycles. Hence, the number of calculations for this reduction is [6]

$$
W=9 Q \frac{a^{2}}{(\Delta x)^{2}} \log \frac{2 a}{\pi \Delta x} / \log R \log \frac{1-R}{1+R} .
$$

As a function of $R, W$ is minimized for $R$ around 0.4 , and for this value of $R$,

$$
W \leq 12 Q a^{2} \log \frac{2 a}{\pi \Delta x} /(\Delta x)^{2}
$$

Experimental evidence indicates that the constant 12 is high and should be about six.

One admissible sequence $a_{n}$ for a general region is that sequence (3.4) for the smallest square containing $D$. If $a$ is the side length of this square and $N(\Delta x)$ is the number of interior lattice points of $D$ corresponding to increment $\Delta x$, then the number of calculations required to reduce the coefficients of the error eigenfunctions by a factor of $\exp (-Q)$ is

$$
W \leq 12 Q N(\Delta x) \log \frac{2 a}{\pi \Delta x}
$$

It is undoubtedly not worth the effort in a practical problem to attempt to obtain a better sequence of iteration parameters.

\section{REFERENCES}

1. J. Douglas, On the numerical integration of $u_{x x}+u_{y y}=u_{t}$ by implicit methods, J. Soc. Ind. Appl. Math. vol. 3 (1955) pp. 42-65.

2. - On the relation between stability and convergence in the numerical solution of linear parabolic and hyperbolic differential equations, Journal of the Society for Industrial and Applied Mathematics vol. 4 (1956) pp. 20-37.

3. J. Douglas and T. M. Gallie, Variable time steps in the solution of the heat flow equation by a difference cquation, Proc. Amer. Math. Soc. vol. 6 (1955) pp. 787-793.

4. J. Douglas, and D. W. Peaceman, Numerical solution of two-dimensional heat flow problems. A.I.Ch.E. Journal vol. 1 (1955) pp. 505-512.

5. W. E. Milne, Numerical solution of differential equations, New York, 1953.

6. D. W. Peaceman, and H. H. Rachford, The numerical solution of parabolic and elliptic differential equations, J. Soc. Ind. Appl. Math. vol. 3 (1955) pp. 28-41.

Humble Oil and Refining Co. 